\title{
Functional Polymers and Sequential Copolymers by Phase Transfer Catalysis VII. Synthesis and Characterization of Alternating Block Copolymers of Aromatic Poly(ether sulfone)s with Aliphatic Polysulfides and Aliphatic Polysulfones
}

\author{
V. Percec, H. Nava, and B. C. Auman \\ Department of Macromolecular Science, \\ Case Western Reserve University, \\ Cleveland, Ohio 44106, U.S.A.
}

(Received February 24, 1984)

\begin{abstract}
Di(bromo)polyhexamethylenesulfides were polyetherified with $\alpha, \omega-$ di(phenol)aromatic poly(ether sulfone)s in the presence of tetrabutylammonium hydrogen sulfate (stoichiometric amounts versus phenolic end groups) as phase transfer catalyst, in a two phase system of $50 \mathrm{wt} \%$ aqueous $\mathrm{NaOH}$ solution and monochlorobenzene. The alternating block copolymers obtained, were transformed into polyhexamethylenesulfone-aromatic poly(ether sulfone) alternating block copolymers by oxidation with $m$-chloroperbenzoic acid in chloroform solution. DSC analysis of both types of alternating block copolymers show phase separation, due to the crystallization of the polyhexamethylenesulfide or polyhexamethylenesulfone blocks.

KEY WORDS Phase Transfer Catalyzed Polyetherification / $\alpha, \omega-$ $\mathrm{Di}$ (bromo)polyhexamethylenesulfide / $\alpha, \omega$-Di(bromo)polyhexamethylenesulfone / $\alpha, \omega$-Di(phenol)aromatic Poly(ether sulfone) / Alternating Block Copolymer / Phase Separation /
\end{abstract}

In several previous papers we have demonstrated that Williamson phase transfer catalyzed polyetherification of $\alpha, \omega$-di(nucleophilic)oligomers with $\alpha, \omega$-di(electrophilic)oligomers can be used as a convenient method for the preparation of alternating block copolymers. ${ }^{1-3}$ The most frequently used $\alpha, \omega$-di(nucleophilic)oligomers were $\alpha, \omega$-di(phenol)aromatic poly(ether sulfone) $\mathrm{s}^{1,2}$ and $\alpha, \omega$-di(phenol)polyisobutylene. ${ }^{3}$ The electrophilic groups which were successfully introduced as the terminal groups of the $\alpha, \omega$-di(electrophilic)oligomers were chloroallyl and bromobenzyl. $^{1-4}$

These $\alpha, \omega$-di(electrophilic)oligomers were prepared by phase transfer catalyzed chain extension of the $\alpha, \omega$-di(phenol)oligomers with cis- or trans-dichloro-2-butene, or $\alpha, \alpha^{\prime}$-dibromo- $p$-xylene.
The same reaction was exploited for the preparation of regular copolymers starting from $\alpha, \omega$-di(nucleophilic)- or $\alpha, \omega$-di(electrophilic)oligomers and either dielectrophilic or dinucleophilic monomers. ${ }^{1,4}$

Recently, we have also shown that ABA triblock copolymers can be conveniently prepared by using a similar synthetic procedure. A poly(2,6-dimethyl-1,4-phenylene oxide) (PPO) with one phenol chain end was quantitatively polyetherified with an $\alpha, \omega$-di(chloroallyl)- or $\quad \alpha, \omega$-di(bromobenzyl)aromatic poly(ether sulfone) (PS) to provide a PPOPS-PPO triblock copolymer. ${ }^{5}$

The major difference between our reaction conditions and the conventional phase transfer catalyzed Williamson etherification ${ }^{6}$ or polyetherification $^{7,8}$ is the use of stoichiometric amounts of phase transfer catalyst versus the 
nucleophilic chain ends in the former case. Under these reaction conditions, both the nucleophilic and electrophilic groups are present in the organic phase. Consequently, the etherification takes place in solution, the reaction is very fast at moderate or even room temperature, and the reaction course can be followed, in some cases, by the disappearance of the green color of the phenolate anion.

The first goal of this paper is to demonstrate that even less reactive electrophilic groups like bromoalkane, can be used as terminal electrophilic groups, suitable for the preparation of alternating block copolymers by phase transfer catalyzed Williamson polyetherification.

The $\alpha, \omega$-di(bromoalkane)oligomers used for this demonstration are aliphatic polysulfides prepared by the phase transfer catalyzed polymerization of 1,6-dibromohexane with 1,6hexanedithiol according to the method developed by Imai et al. ${ }^{9} \alpha, \omega-\mathrm{Di}($ phenol)aro- matic polyether sulfones (PS) were used as $\alpha, \omega$-di(nucleophilic)oligomers.

The second goal of this paper is to present the synthesis and characterization of two novel alternating block copolymers. The first is an alternating block copolymer of an aliphatic polysulfide with an aromatic polyether sulfone. Previously, aliphatic polysulfide containing block copolymers were prepared only by living anionic polymerization of cyclic sulfides. A recent book compiles all these block copolymers. ${ }^{10}$ The limitations of anionic polymerization for the preparation of aliphatic polysulfide containing block copolymers consist of the following. There are a limited number of cyclic sulfides which do polymerize through a living mechanism (ethylene sulfide, propylene sulfide, isobutylene sulfide) and their synthesis is not very convenient. The nature of the second block is limited to monomers which also polymerize through a living anionic mechanism. It also does not provide any avenue for the

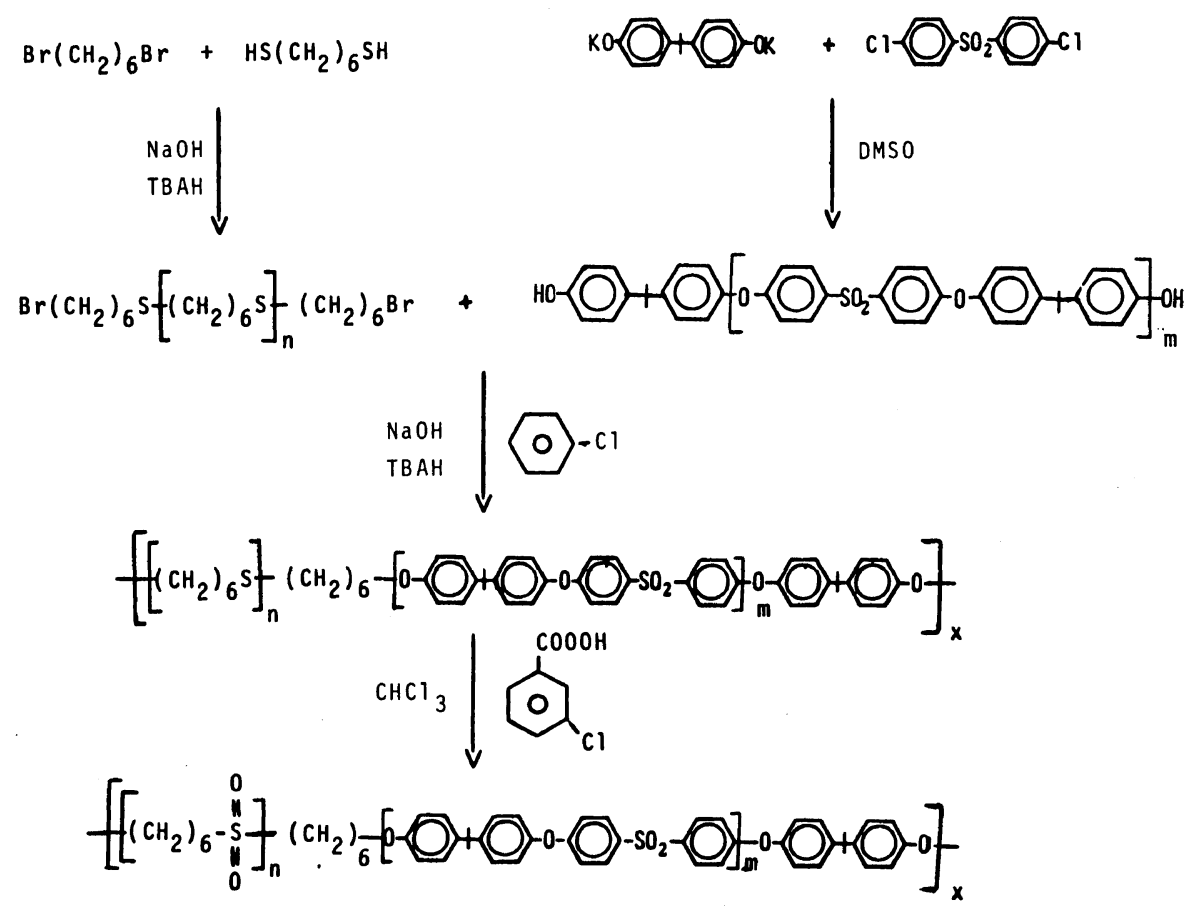

Scheme 1. Synthesis of alternating block copolymers of aromatic poly(ether sulfone)s with aliphatic polysulfides and aliphatic polysulfones. 
preparation of alternating block copolymers.

The advantage of the method presented here is that it can basically use any polysulfide which can be prepared by phase transfer catalyzed condensation of an $\alpha, \omega$-di(bromo)alkane with an $\alpha, \omega$-di(thio)alkane as one block and any $\alpha, \omega$-di(phenol)oligomer as the other. The second novel alternating block copolymer consists of an aliphatic polysulfone with an aromatic polyether sulfone. It was prepared through the oxidation of the thioether linkages in the first block copolymer with $m$ chloroperbenzoic acid in chloroform solution. Both types of alternating block copolymers present phase separation due to the crystallization of the aliphatic polysulfide or aliphatic polysulfone blocks. Scheme 1 outlines the synthetic routes used for the preparation of both types of block copolymers.

\section{EXPERIMENTAL}

\section{A. Materials}

Bisphenol-A (BPA) (Aldrich) was recrystallized from aqueous methanol. 4,4'-Dichlorodiphenyl sulfone (DCPS) (Aldrich) was recrystallized twice from toluene. 1,6-Dibromohexane, 1,6-hexanedithiol, tetrabutylammonium hydrogen sulfate (TBAH), monochlorobenzene $(\mathrm{ClBz})$, as well as, the other solvents (all from Aldrich) were used as received.

\section{B. Techniques}

$60 \mathrm{MHz}{ }^{1} \mathrm{H}$ NMR spectra were recorded on Varian A-60 and EM-360 instruments. $200 \mathrm{MHz}{ }^{1} \mathrm{H}$ NMR spectra were recorded on a Varian XL-200 spectrometer. All spectra were obtained from $\mathrm{CDCl}_{3}$ solutions and with TMS as internal standard. A Digilab FTIR spectrometer was used to record IR spectra of polymer films on $\mathrm{KBr}$ plates or of polymer powders in $\mathrm{KBr}$ pellets. DSC curves, glass transition temperature $\left(T_{\mathrm{g}}\right)$, melting temperature $\left(T_{\mathrm{m}}\right)$, and crystallization temperatures $\left(T_{\mathrm{c}}\right)$ were determined by a Perkin-Elmer Differ- ential Scanning Calorimeter (model DSC-2). Indium was used as calibration standard. The $T_{\mathrm{g}}$ values of $\alpha, \omega$-di(phenol)PS were recorded during the second heating cycle. GPC analyses were carried out with IR and UV detectors using $\mu$-Styragel columns of $10^{4}$ and $10^{3} \AA$ and a calibration plot constructed with polystyrene standards.

C. Synthesis of $\alpha, \omega-D i($ phenol)aromatic Poly(ether sulfone)s (PS)

The $\alpha, \omega$-di(phenol)PS oligomers were prepared by the condensation of excess potassium salt of BPA with DCPS in anhydrous DMSO. Their detailed synthesis and characterization were presented in a previous paper, ${ }^{1}$ and their $M_{n}$ and $T_{\mathrm{g}}$ values are presented in Table I.

D. Synthesis of $\alpha, \omega-D i$ (bromo)poly(hexamethylene sulfide) (PHSU)

A mixture of $1.058 \mathrm{~g}(7.053 \mathrm{mmol})$ of $1,6-$ hexanedithiol, $1.7209 \mathrm{~g}(7.053 \mathrm{mmol})$ of 1,6 dibromohexane, $0.0454 \mathrm{~g} \quad(0.141 \mathrm{mmol})$ of TBAH and $14 \mathrm{ml}$ of $1.0 \mathrm{~N}$ aqueous potassium hydroxide was vigorously stirred at $80^{\circ} \mathrm{C}$ for $24 \mathrm{~h}$. The polymer gradually separated in the form of molten mass as the polymerization proceeded. After the reaction mixture was cooled the solidified polymer was separated, dissolved in chloroform and precipitated twice into methanol, filtered and dried under vacuum at room temperature. Yield, 98\%.

\section{E. Synthesis of $\alpha, \omega-D i($ bromo)poly(hexa- methylene sulfone) (PHSO)}

PHSO was prepared through the oxidation of PHSU according to a method developed by Marvel. ${ }^{11}$ The only difference was that $m$ chloroperbenzoic acid was used as an oxidating agent instead of perbenzoic acid. A solution of $1.16 \mathrm{~g}\left(M_{n}=1590,0.01 \mathrm{~mol}\right.$ of repeating units) of PHSU was dissolved in $50 \mathrm{ml}$ of chloroform and cooled to $-5^{\circ} \mathrm{C}$. $4.66 \mathrm{~g}$ $(0.027 \mathrm{~mol})$ of $m$-chloroperbenzoic acid dissolved in $50 \mathrm{ml}$ chloroform and cooled to $10^{\circ} \mathrm{C}$ was added to the polymer solution. A white 
precipitate started to form as soon as the $m$ chloroperbenzoic acid was added. The reaction mixture was stirred at $-5^{\circ} \mathrm{C}$ for $2 \mathrm{~h}$ and at $10^{\circ} \mathrm{C}$ overnight. The precipitate was filtered, then washed with acetone, methanol, aqueous $\mathrm{NaHCO}_{3}$, again with methanol and then dried under vacuum at room temperature. Yield, $90 \%$.

\section{F. Synthesis of PS-PHSU Alternating Block} Copolymers

The general procedure used for the preparation of PS-PHSU alternating block copolymers is presented in the following example. To a stirring solution of $0.5 \mathrm{~g}\left(M_{n}=3,875\right.$; $0.129 \mathrm{mmol}$ ) of PS-4 in $3 \mathrm{ml}$ monochlorobenzene were added $0.05 \mathrm{ml}$ of a $50 \% \mathrm{NaOH}$ water solution. The sodium salt of PS precipitated immediately. After the addition of $0.0876 \mathrm{~g}$ $(0.258 \mathrm{mmol})$ of TBAH, the reaction mixture returned to a clear solution. $\alpha, \omega-\mathrm{Di}-$ (bromo)PHSU $\quad\left(0.205 \mathrm{~g}, \quad M_{n}=1,590,0.129\right.$ mmol) was added and the reaction was allowed to stir at $70^{\circ} \mathrm{C}$ for $24 \mathrm{~h}$. After cooling, the reaction mixture was washed several times with water, and then was precipitated into methanol. A second purification was done by precipitating a filtered chloroform solution of the resulting block copolymer in methanol. The yield was higher than $95 \%$ in all cases.

G. Synthesis of PS-PHSO Alternating Block Copolymers

These were prepared by using the same procedure as for the preparation of the PHSO.

\section{RESULTS AND DISCUSSION}

Synthesis and Structural Characterization of PS-PHSU and PS-PHSO Alternating Block Copolymers

$\alpha, \omega$-Di(bromo)PHSU was prepared according to the method previously reported by Imai et al. ${ }^{9}$ The only difference was that we used TBAH as a phase transfer catalyst. A typical $200 \mathrm{MHz}{ }^{1} \mathrm{H}$ NMR spectrum of the $\alpha, \omega$ di(bromo)PHSU is presented in Figure 1. The protonic resonances in this figure were assigned using the $200 \mathrm{MHz}{ }^{1} \mathrm{H}$ NMR spectra of 1,6-dibromohexane (Figure 2) and 1,6-hexanedithiol (Figure 3) as model compounds for

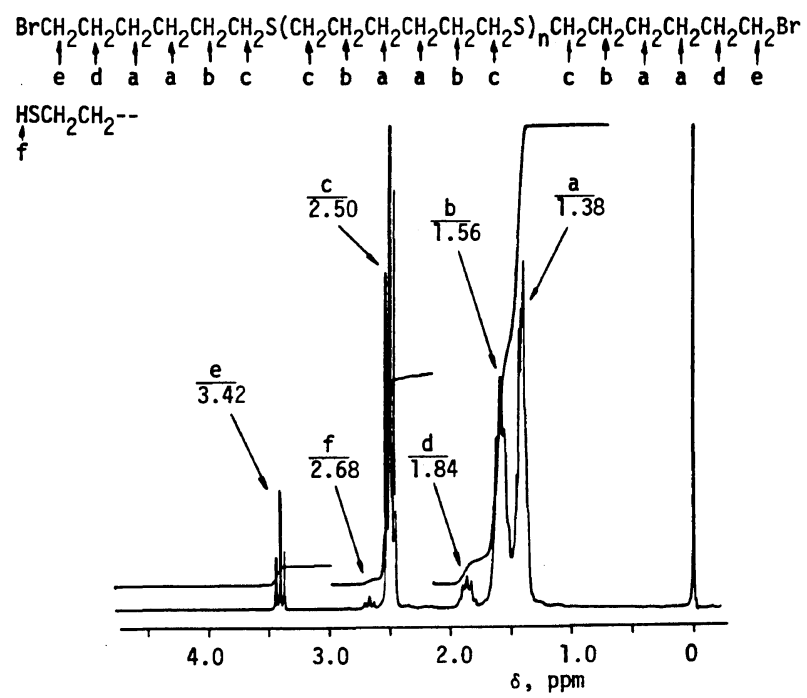

Figure 1. $200 \mathrm{MHz}{ }^{1} \mathrm{H}$ NMR spectrum (CDCl 3 , TMS) of $\alpha, \omega$-di(bromo)PHSU (sample PHSU-1 from Table I). 
Functional Polymers and Sequential Copolymers by Phase Transfer Catalysis VII.

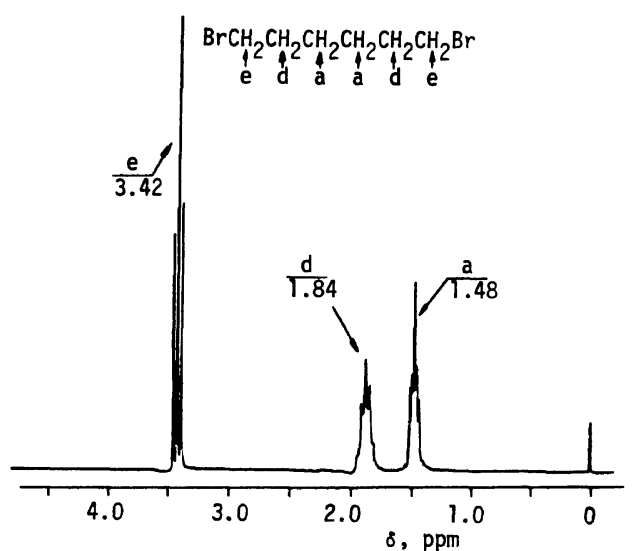

Figure 2. $200 \mathrm{MHz}{ }^{1} \mathrm{H}$ NMR spectrum $\left(\mathrm{CDCl}_{3}, \mathrm{TMS}\right)$ of 1,6-dibromohexane.

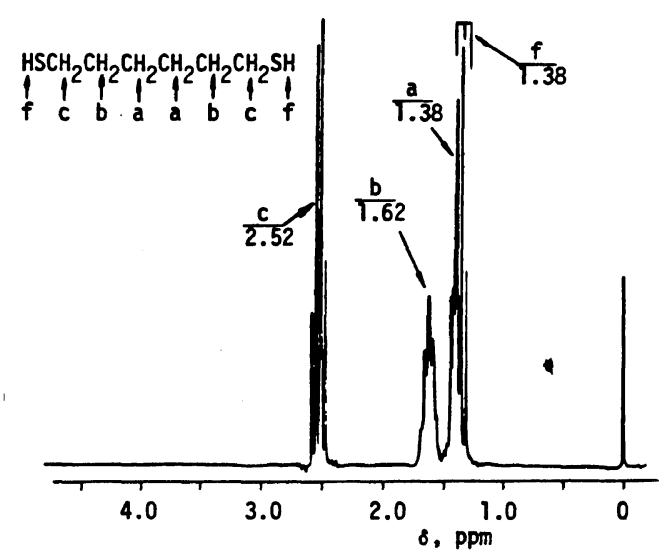

Figure 3. $200 \mathrm{MHz}^{1} \mathrm{H}$ NMR spectrum $\left(\mathrm{CDCl}_{3}, \mathrm{TMS}\right)$ of 1,6-hexanedithiol.

the $\alpha, \omega$-di(bromo)PHSU chain ends and internal structural units, respectively. The presence of the triplet at $3.42 \mathrm{ppm}$ due to $\mathrm{BrCH}_{2}$ (signal e in Figure 1) and of the multiplet at $1.84 \mathrm{ppm}$ due to the $-\mathrm{CH}_{2}-$ groups next to the $\mathrm{BrCH}_{2}-$ groups (signal d in Figure 1) demonstrates that the $\alpha, \omega$-di(bromo)PHSU contains bromine chain ends. $60 \mathrm{MHz}{ }^{1} \mathrm{H} \mathrm{NMR}$ spectroscopy was not enough sensitive to analyze the strucrure of this polymer's chain ends. The resonance of the methylene groups vicinal to thioether bond appears as a triplet at $2.5 \mathrm{ppm}$ (signal c in Figure 1). The next methylene groups from the $-\mathrm{CH}_{2}-\mathrm{S}-\mathrm{CH}_{2}-$ group appear at $1.56 \mathrm{ppm}$ (signal b), while the two internal methylene groups appear at $1.38 \mathrm{ppm}$ (signal a, Figure 1). The FTIR spectrum of this polymer does not show any absorption for the $-\mathrm{SH}$ stretching at $2550 \mathrm{~cm}^{-1}$. In spite of this, the ${ }^{1} \mathrm{H}$ NMR spectrum in Figure 1 shows an unexpected triplet at $2.68 \mathrm{ppm}$ (signal f). Several polymers prepared under the same reaction conditions did show the presence of this signal even after a very rigorous purification by successive reprecipitations from chloroform into methanol or hexane.

In a previous paper we demonstrated that in the absence of a stronger nucleophile than $\mathrm{OH}^{-}$, under phase transfer catalyzed reactions the nucleophilic substitution of $-\mathrm{Cl}$ or $-\mathrm{Br}$ with $-\mathrm{OH}$ takes place. ${ }^{13}$ If this reaction occurs in the present case, then we would expect to see a triplet due to $-\mathrm{CH}_{2} \mathrm{OH}$ or even due to $-\mathrm{CH}_{2}-$ $\mathrm{O}-\mathrm{CH}_{2}-$ (which can be obtained by the etherification of the $-\mathrm{CH}_{2} \mathrm{OH}$ with $-\mathrm{CH}_{2} \mathrm{Br}$ chain ends) groups at about $3.55 \mathrm{ppm}$ or even at lower field. The spectrum in Figure 1 does not show any signal in this region. Consequently, we can speculate that the triplet at $2.68 \mathrm{ppm}$ can be due to a very small concentration of thiol $\left(\mathrm{HSCH}_{2}-\right)$ chain ends. Very small concentrations of HS- groups could not be detected even by FTIR because the -SH stretching at $2550 \mathrm{~cm}^{-1}$ is a very weak absorption. Presently, we do not have any other support for this assumption. But if the signal at $2.68 \mathrm{ppm}$ is correctly assigned, then the presence of a small concentration of - $\mathrm{SH}$ groups will not affect the alternating block copolymerization, because by polyetherification they will give rise to chain extension.

The degree of polymerization of $\alpha, \omega$ di(bromo)PHSU was determined from $200 \mathrm{MHz}{ }^{1} \mathrm{H}$ NMR integrations by using the structural formula depicted on Figure 1 and using the following relationships:

$$
\begin{aligned}
\overline{\mathrm{DP}} & =[\mathrm{Aa} / 4] /[\mathrm{Ae} / 4]-2 \\
& =[\mathrm{Ab} / 4] /[\mathrm{Ae} / 4]-1 \\
& =[\mathrm{Ac} / 4] /[\mathrm{Ae} / 4]-1
\end{aligned}
$$




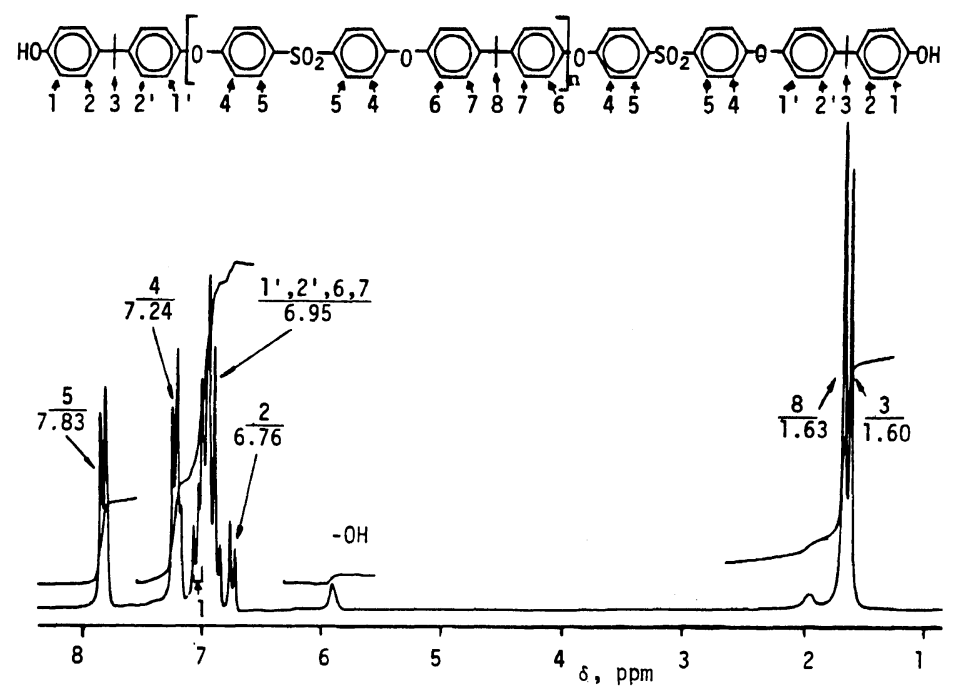

Figure 4. $200 \mathrm{MHz}^{1} \mathrm{H}$ NMR spectrum ( $\mathrm{CDCl}_{3}, \mathrm{TMS}$ ) of $\alpha, \omega$-di(phenol)PS (sample PS-5 from Table I).

The $M_{n}$ values for two samples prepared under identical reaction conditions are presented in Table I. $60 \mathrm{MHz}{ }^{1} \mathrm{H}$ NMR spectroscopy is not powerful enough to detect the polymer chain ends, and consequently, it can not be used for the $M_{n}$ determinations.

Figure 4 presents a typical $200 \mathrm{MHz}{ }^{1} \mathrm{H}$ NMR spectrum of an $\alpha, \omega$-di(phenol)PS, while Figure 5 shows a typical $200 \mathrm{MHz}{ }^{1} \mathrm{H}$ NMR spectrum of a PS-PHSU alternating block copolymer (sample PS-2-PHSU-2 from Table I). The protonic assignments are shown on both figures. It is important to notice that the signal e is shifted from $3.42 \mathrm{ppm}$ in Figure 1 $\left(-\mathrm{CH}_{2} \mathrm{Br}\right)$ to $3.9 \mathrm{ppm}$ in Figure $5\left(-\mathrm{CH}_{2}-\mathrm{O}-\right.$ $\mathrm{Ph})$. This demonstrates that all the bromoalkyl chain ends of the $\alpha, \omega$-di(bromo)HSU have reacted with the phenol chain ends of the $\alpha, \omega$ di(phenol)PS. The only other significant difference between the spectrum of the alternating block copolymer in Figure 5 and the spectra of the starting materials from Figures 1 and 4 is that signal 1 [due to ortho phenyl protons (versus- $\mathrm{OH}$ ) from the phenol end groups of PS] (Figure 4) was shifted downfield after polyetherification (Figure 5).

The alternating block copolymers were also

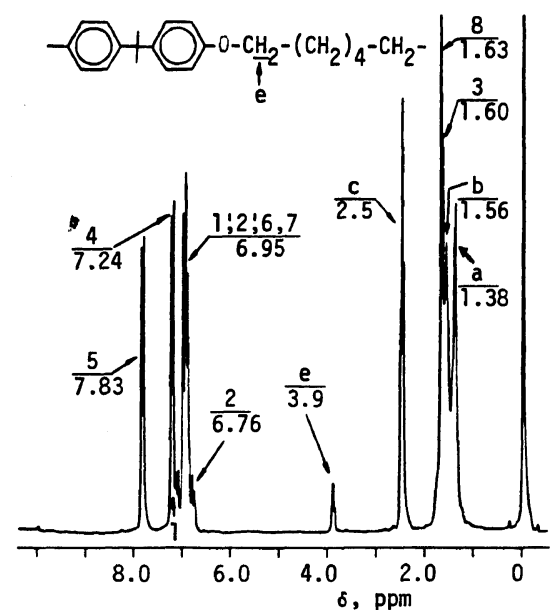

Figure 5. $200 \mathrm{MHz}^{1} \mathrm{H}$ NMR spectrum $\left(\mathrm{CDCl}_{3}, \mathrm{TMS}\right)$ of PS-PHSU alternating block copolymer (sample PS-2PHSU-2, No. 3 from Table I).

characterized by GPC analyses. Figure 6 shows typical GPC curves for $\alpha, \omega$ di(bromo)PHSU (a, PHSU-2 $M_{n}=1,403$ from Table I), $\alpha, \omega$-di(phenol)PS (b, PS-2, $M_{n}=$ 3,050 from Table I) and for the obtained alternating block copolymer (c, Sample-3 from Table I). In all cases, the GPC traces of the block copolymers show a small tail at high 
Functional Polymers and Sequential Copolymers by Phase Transfer Catalysis VII.

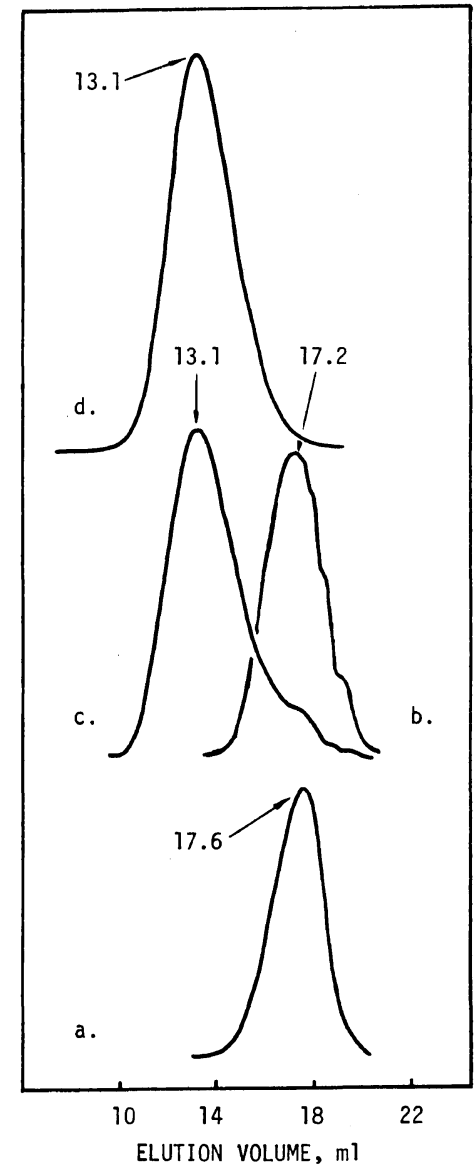

Figure 6. Gel Permeation Chromatograms of: a) $\alpha, \omega$ di(bromo)PHSU-2; b) $\alpha, \omega$-di(phenol)PS-2; c) unpurified PS-2-PHSU-2 alternating block copolymer (sample No. 3 from Table I); d) alternating block copolymer PS-2PHSU-2 purified by precipitation with acetonemethanol $(1: 3, \mathrm{v} / \mathrm{v})$.

elution volume (Figure 6c). It could be removed by the precipitation of the block copolymer from chloroform with a mixture of acetone-methanol $(1: 3, \mathrm{v} / \mathrm{v})$. A GPC trace for a purified block copolymer is shown in Figure $6 \mathrm{~d}$.

The degrees of polymerization $(n)$ of the alternating block copolymers (PS-PHSU) were determined by GPC analysis and are presented in Table I. Their values range between 2 and 4 when determined from $M_{n}$ and between 5 and 8 when determined from $M_{w}$.

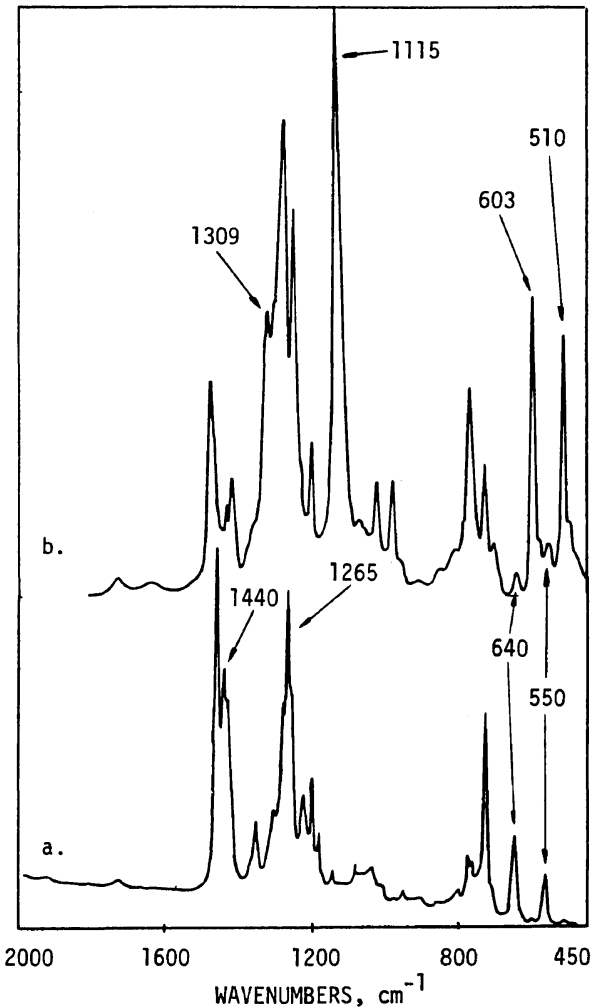

Figure 7. FT-IR spectra of: a) $\alpha, \omega$-di(bromo)PHSU-1 (film on $\mathrm{KBr}$ plate); b) $\alpha, \omega$-di(bromo)PHSO-1 $(\mathrm{KBr}$ pellet).

It is well known that aliphatic polysulfides can be transformed into aliphatic polysulfones by oxidation. ${ }^{11}$ Suitable reaction conditions can provide almost complete oxidation of polysulfides to polysulfones. The obtained $\alpha, \omega$-di(bromo)PHSO is insoluble in most common organic solvents, ${ }^{12}$ and consequently it was characterized only by FTIR. The FTIR spectrum of the $\alpha, \omega$-di(bromo)PHSU (Figure 7a) presents the following characteristic absorptions: 550 and $640 \mathrm{~cm}^{-1}$ (C-S stretching), $1265 \mathrm{~cm}^{-1}$ (C-S wagging) and $1440 \mathrm{~cm}^{-1}$ (C-S deformation). After oxidation (Figure 7b) its spectrum no longer presents the ab: sorption at $1440 \mathrm{~cm}^{-1}$, but it still shows very weak absorptions at 550 and $640 \mathrm{~cm}^{-1}$. This demonstrates that not all the sulfide groups were oxidized. In addition the FTIR spec- 
Table I. Synthesis and characterization of the alternating block copolymers of PS with PHSU and PHSO

\begin{tabular}{|c|c|c|c|c|c|c|c|c|c|c|c|c|c|c|c|c|c|c|c|}
\hline \multirow{3}{*}{ No. } & \multicolumn{6}{|c|}{$\alpha, \omega$-Di(phenol)PS } & \multicolumn{7}{|c|}{$\alpha, \omega$-Di(bromo)PHSU } & \multicolumn{6}{|c|}{$(\text { PS-PHSU })_{n}$} \\
\hline & \multirow{2}{*}{ Sample } & \multirow{2}{*}{$\begin{array}{c}M_{n} \\
\text { NMR }\end{array}$} & \multirow[t]{2}{*}{$M_{n}$} & \multirow{2}{*}{$\frac{M_{w}}{\text { GPC }}$} & \multirow[t]{2}{*}{$M_{w} / M_{n}$} & \multirow{2}{*}{$\frac{T_{\mathrm{g}}}{{ }^{\circ} \mathrm{C}}$} & \multirow{2}{*}{ Sample } & \multirow{2}{*}{$\begin{array}{c}M_{n} \\
\text { NMR }\end{array}$} & \multirow[t]{2}{*}{$M_{n}$} & \multirow{2}{*}{$\frac{M_{w}}{\text { GPC }}$} & \multirow{2}{*}{$M_{w} / M_{n}$} & \multirow{2}{*}{$T_{\mathrm{m}}$} & \multirow[t]{2}{*}{$T_{\mathrm{c}}$} & \multirow[t]{2}{*}{$M_{n}$} & \multirow{2}{*}{$\frac{M_{w}}{\text { GPC }}$} & \multirow{2}{*}{$M_{w} / M_{n}$} & \multicolumn{2}{|c|}{$n$ by } & \multirow{2}{*}{$\begin{array}{c}T_{\mathrm{m}} \\
{ }^{\circ} \mathrm{C}\end{array}$} \\
\hline & & & & & & & & & & & & & & & & & $M_{n}$ & $M_{w}$ & \\
\hline 1. & PS-1 & 2.550 & 2,343 & 2,935 & 1.3 & 135 & PHSU-1 & 1,590 & 2,308 & 3,186 & 1.4 & 64.0 & 45.0 & 15,980 & 49.295 & 3.1 & 3.4 & 8.1 & 70.5 \\
\hline 2. & - & - & - & - & - & - & PHSO- $1^{a}$ & - & - & - & - & $\begin{array}{l}188.5 \\
196.5\end{array}$ & 170.0 & - & - & - & 一 & - & - \\
\hline 3. & PS-2 & 3,050 & 2,820 & 4,411 & 1.6 & 138 & PHSU-2 & 1,403 & 2,128 & 2,749 & 1.3 & 65.0 & 51.5 & 16,196 & 33,887 & 2.1 & 3.3 & 4.7 & 46.0 \\
\hline 4. & PS-2 & 3,050 & 2,820 & 4,411 & 1.6 & 138 & PHSO-2 ${ }^{b}$ & - & - & - & - & - & - & - & - & - & - & - & $\begin{array}{l}146.0^{\mathrm{c}} \\
183.0\end{array}$ \\
\hline 5. & PS-3 & 3,410 & 3,269 & 5,566 & 1.7 & 148 & PHSU-1 & 1,590 & 2,308 & 3,186 & 1.4 & 64.0 & 45.0 & 16,711 & 59,080 & 3.5 & 3.0 & 6.8 & 65.5 \\
\hline 6. & PS-4 & 3,875 & 3,409 & 5,835 & 1.7 & 146 & PHSU-1 & 1,590 & 2,308 & 3,186 & 1.4 & 64.0 & 45.0 & 14,381 & 51,867 & 3.6 & 2.5 & 5.8 & 65.0 \\
\hline 7. & PS-5 & 1,210 & - & - & - & 87 & PHSU-1 & 1,590 & 2,308 & 3,186 & 1.4 & 64.0 & 45.0 & 11,782 & 32,149 & 2.7 & - & - & 49.0 \\
\hline
\end{tabular}

a Prepared by the oxidation of PHSU-1.

b Prepared by the oxidation of (PS-2-PHSU-2)

c $T_{\mathrm{c}}=139.5^{\circ} \mathrm{C}$ 


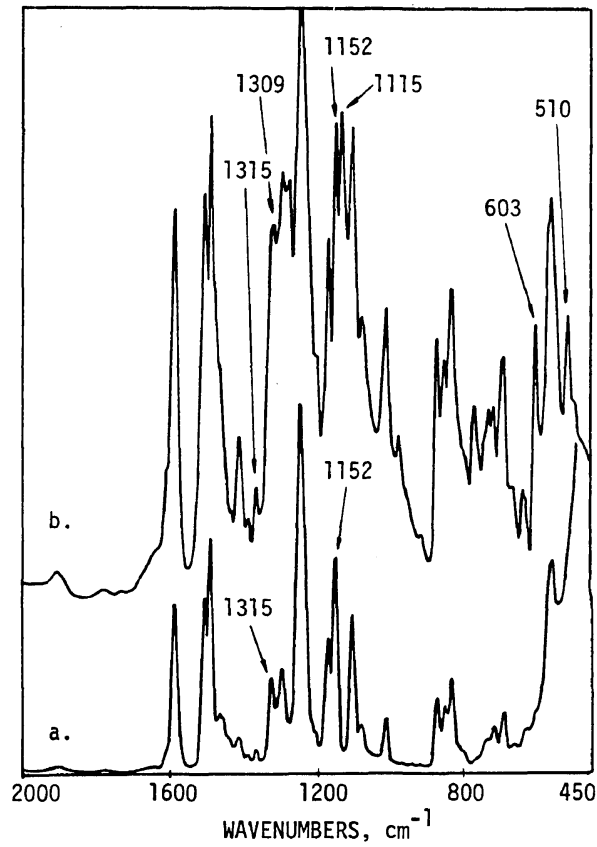

Figure 8. FT-IR spectra of: a) PS-2-PHSU-2 alternating block copolymer (sample No. 3 from Table I, film on $\mathrm{KBr}$ Plate); b) PS-2-PHSO-2 alternating block copolymer (sample No. 4 from Table I, KBr pellet).

trum in Figure $7 \mathrm{~b}$ presents the characteristic absorptions of the aliphatic sulfone group: $510 \mathrm{~cm}^{-1}$ (-SO $\mathrm{SO}_{2}$ - wagging), $603 \mathrm{~cm}^{-1}\left(-\mathrm{SO}_{2}-\right.$ scissors), $1115 \mathrm{~cm}^{-1}\left(v-\mathrm{SO}_{2}-\mathrm{sym}\right)$ and 1309 $\mathrm{cm}^{-1}\left(v-\mathrm{SO}_{2}\right.$-assym).

The oxidation of the PS-PHSU (Block No. 3 from Table I) alternating block copolymer provided a simple avenue for the preparation of an alternating block copolymer of PS and PHSO (Block No. 4 in Table I). The PS-PHSO alternating block copolymer is insoluble in common organic solvents as was the HPSO, and consequently, it was characterized only by FTIR spectroscopy (Figure 8). Attempts to extract unreacted $\alpha, \omega$-di(phenol)PS from this insoluble block copolymer (with solvents specific to PS like $\mathrm{CHCl}_{3}, \mathrm{CH}_{2} \mathrm{Cl}_{2}$, etc.) failed. This is additional evidence that the alternating block copolymers prepared by polyetherification (once purified by precipitation in acetone-methanol $=1: 3, \mathrm{v} / \mathrm{v}$ ) are free of unre- acted materials. The FTIR spectrum of the PS-PHSU block copolymer shows the characteristic absorptions for aromatic sulfone groups at $1152 \mathrm{~cm}^{-1}\left(v-\mathrm{SO}_{2}-\mathrm{sym}\right)$ and 1315 $\mathrm{cm}^{-1}\left(v-\mathrm{SO}_{2}\right.$-assym) (Figure $\left.8 \mathrm{a}\right)$. After oxidation, it also presents the absorptions characteristic to aliphatic sulfone group at: $510 \mathrm{~cm}^{-1}$ (-SO $\mathrm{S}_{2}$-wagging), $603 \mathrm{~cm}^{-1}$ (-SO $\mathrm{SO}_{2}$-scissors), $1115 \mathrm{~cm}^{-1}\left(\nu-\mathrm{SO}_{2}-\mathrm{sym}\right)$ and $1309 \mathrm{~cm}^{-1}(\nu-$ $\mathrm{SO}_{2}$-assym).

Thermal Characterization of PS-PHSU and PS-PHSO Alternating Block Copolymers

Aromatic polyether sulfones are amorphous, and the $T_{\mathrm{g}}$ values of the initial $\alpha, \omega$ di(phenol)PS are presented in Table I. Aliphatic polysulfides are crystalline and the melting, as well as, the crystallization temperatures of the $\alpha, \omega$-di(bromo)PHSU were determined by DSC and are listed in Table I. Typical DSC traces of the PHSU-1 are shown in Figure 9. The heating run (a) presents a melting at $64^{\circ} \mathrm{C}$ and the cooling run (b) a crystallization at $45^{\circ} \mathrm{C}$. We could not detect the $T_{\mathrm{g}}$ of the PHSU in the range of temperatures from $-70^{\circ} \mathrm{C}$ to above the melting temperature. This is not unexpected for highly crystalline polysulfides. ${ }^{14}$ On the other hand, we did expect to observe domain formation in the PS-PHSU block copolymers due to the crystallization of the PHSU blocks. This is the general behavior of polysulfides containing block copolymers. ${ }^{15}$ All the DSC analyzes carried out on PS-PHSU alternating block copolymers were done after the sample was purified by precipitation from chloroform solution into acetone-methanol $(1: 3, \mathrm{v} / \mathrm{v})$. A DSC curve for the PS-PHSU block copolymer No. 3 from Table I is presented in Figure 9c. It shows the melting of the PHSU blocks and demonstrates the phase separation in these block copolymers. The melting temperatures of the PS-PHSU block copolymers are presented in Table I and are slightly different from those of the parent materials. It is interesting to notice that we could not find any $T_{\mathrm{g}}$ for 


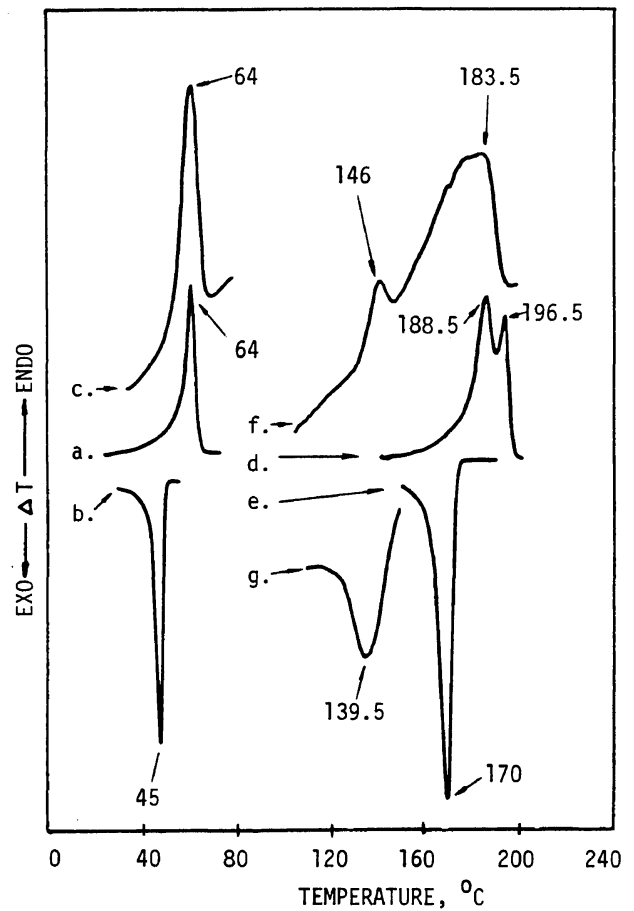

Figure 9. DSC curves of: a) $\alpha, \omega$-di(bromo)PHSU-1 (heating scan); b) $\alpha, \omega$-di(bromo)PHSU-1 (cooling scan); c) PS-2-PHSU-2 (sample No. 3 from Table I) alternating block copolymer (heating scan); d) $\alpha, \omega$-di(bromo)PHSO-1 (heating scan); e) $\alpha, \omega$-di(bromo)PHSO-1 (cooling scan); f) PS-2-PHSO-2 alternating block copolymer (sample No. 4 from Table I, heating scan); g) PS-2-PHSO-2 alternating block copolymer (sample No. 4 from Table I, cooling scan).

these block copolymers. At the same time, on cooling, the DSC curves of PS-PHSU block copolymers do not show any crystallization of the PHSU blocks. This is because the crystallization of the PHSU blocks takes place below the $T_{\mathrm{g}}$ values of the PS blocks, and consequently would require long annealing. The crystallization of the PHSU blocks occurs easily by casting PS-PHSU films from $\mathrm{CHCl}_{3}$ solution.

Typical DSC curves of the PHSO-1 are shown in Figure 9 (d, e). On heating (Figure 9d), this polymer shows two melting points at 188.5 and $196.5^{\circ} \mathrm{C}$, respectively. We can assume that the two meltings are due to the heterogeneous microstructure of the PHSO blocks (incomplete oxidation of PHSU). On cooling (Figure 9e), we can see only a sharp crystallization at $170^{\circ} \mathrm{C}$. The DSC curve of PS-PHSO block copolymers (Block 4 from Table I) shows both the two meltings on heating (Figure 9f) at 146 and $183^{\circ} \mathrm{C}$ (incomplete oxidation of the PHSU segments), and also the crystallization on cooling (Figure 9g) at $139.5^{\circ} \mathrm{C}$. The crystallization of the PHSO blocks on cooling is in contrast to the behavior of the PS-PHSU block copolymers, but it is at the same time an expected occurrence. This is because the crystallization of the PHSO blocks occurs above the $T_{\mathrm{g}}$ values of the PS block. As we can see from Table I, the $\alpha, \omega$-di(phenol)PS initially used for the preparation of this block copolymer has a $T_{\mathrm{g}}$ of $138^{\circ} \mathrm{C}$ which is not far below the crystallization temperature of the PHSO blocks $\left(139.5^{\circ} \mathrm{C}\right)$. Previously,${ }^{16,17}$ we have shown that $\alpha, \omega$-di(phenol)PS oligomers present $T_{\mathrm{g}}$ values which are up to $31^{\circ} \mathrm{C}$ higher than those of the corresponding polymers endcapped with groups which do not give rise to hydrogen bonding. For example, the $T_{\mathrm{g}}$ value of the $\alpha, \omega$-di(phenol)PS- 2 is $138^{\circ} \mathrm{C}$ (Table I) while after etherification with an inert functional group, it drops to $117^{\circ} \mathrm{C}$. Consequently, the actual $T_{\mathrm{g}}$ of the PS block in PS-PHSO block copolymer should be in this range of temperatures, i.e., far enough from the crystallization temperature $\left(139.5^{\circ} \mathrm{C}\right)$, and consequently, the crystallization of PHSO blocks occurs very fast.

PS-PHSO alternating block copolymers are insoluble and present a high melting temperature for the PHSO blocks. Based on this they may find interesting applications. We know that in certain applications the weak point of conventional aromatic polyether sulfones is that they undergo solvent-induced stress crazing and cracking due to their high sensitivity toward a large variety of solvents. ${ }^{18}$ A PS-PHSO block copolymer would be far superior from this point of view but it is insoluble and can not be cast as a film. Prèsent- 
ly, we are investigating the heterophase phase transfer catalyzed oxidation of PS-PHSU films to provide a simple alternative i.e., the surface oxidation of PS-PHSU films.

Acknowledgement. Financial support from National Science Foundation is gratefully acknowledged.

\section{REFERENCES}

1. V. Percec and B. C. Auman, Makromol. Chem., 185, 617 (1984).

2. V. Percec, B. C. Auman and P. L. Rinaldi, Polym. Bull., 10, 391 (1983).

3. V. Percec, B. C. Auman and J. P. Kennedy, to be submitted.

4. V. Percec and B. C. Auman, Polym. Bull., 10, 385 (1983).

5. V. Percec and H. Nava, Makromol. Chem. Rapid Commun., 5, 319 (1984).

6. H. H. Freeman and R. A. Dubois, Tetrahedron Lett., 38, 3251 (1975).
7. Y. Imai, J. Macromol. Sci. Chem., A15(5), 833 (1981).

8. N. Yamazaki and Y. Imai, Polym. J., 15, 603 (1983).

9. Y. Imai, A. Kato, M. Li and M. Ueda, J. Polym. Sci., Polym. Lett. Ed., 17, 579 (1979).

10. M. Morton, "Anionic Polymerization: Principles and Practice," Academic Press, New York, N.Y., 1983, p 179.

11. C. S. Marvel and P. H. Aldrich, J. Am. Chem. Soc., 72, 1978 (1950).

12. D. W. Ovenall, R. S. Sudol and G. A. Cabat, J. Polym. Sci., Polym. Chem. Ed., 11, 233 (1973).

13. V. Percec and P. L. Rinaldi, Polym. Bull., 10, 233 (1983).

14. R. S. Nevin and E. M. Pearce, J. Polym. Sci., B, 3, 487 (1965).

15. M. Morton and S. L. Mikesell, J. Macromol. Sci., Chem., A7, 1391 (1973).

16. V. Percec and B. C. Auman, Makromol. Chem., 185 (1984) in press.

17. V. Percec and B. C. Auman, Polym. Prepr. Am. Chem. Soc. Div. Polym. Chem., 25(1), 122 (1984).

18. P. M. Hergenrother, J. Polym. Sci., Polym. Chem. Ed., 20, 3131 (1982). 